\title{
Starting of shipboard ESG monitors at low latitudes
}

\author{
Zhi Liu, Jian Zhou, Hang Liu, Jun Yan
}

China Satellite Maritime Tracking and Control Department, Jiangyin, 214431, China

\begin{abstract}
When you start from the prone ESG monitor low latitudes delayed by six schools and cannot be calibrated by two questions, we analyze the reasons for the constraints and six school and calibration phase, obtained ESG can monitor calibration No normal with the latitude and elevation angle of peak to peak on the gyro about the conclusions emerging ESG monitor when running at low latitudes for the problem of excessive and gyro calibration factor uncaught trap questions with appropriate measures for the future electrostatic gyro monitor startup at low latitudes to provide a reference.
\end{abstract}

Keywords: tracking ship; ESG monitor; Low latitudes; Calibration.

\section{Introduction}

ESG monitor the characteristics of navigation information is high-precision, high stability and weather, but also has a long start-up time, complex operation, during startup of offshore sailing conditions require high disadvantage, and is currently static in $5^{\circ} \mathrm{N} \sim 5^{\circ} \mathrm{S}$ Sea ( referred to low latitudes) start success rate is not high, full of static electricity utility, to explore up and running policy-static low-latitude regions, to develop a reasonable electrostatic lower latitudes science starts a workflow based on tracking ship sailing programs to improve efficiency in the use of static electricity, to compensate for weather conditions theodolite star large dependence can not provide real-time shortage heading difference data, give full play to the initial electrostatic heading zero stable, long small navigation error change during the course of the advantages of their positions to further improve the tracking ship boat pose course measurement accuracy. The ship is currently static start occurred at low latitudes delayed by six schools and calibration issues can not, so how to solve this problem better play the role of static electricity in the survey vessel monitoring and control tasks in the focus of this study.

\section{Problem analysis}

\subsection{Analysis of six school stage}

Electrostatic six calibrations aimed at measuring the vertical angle of the initial position of the gyroscope, in order to ensure the accuracy of the initial position angle measurement system to strictly control the parameters of the measurement process error, and the error by setting the scope to control six calibrations workflow.

System error constraint is:

$$
\begin{aligned}
& \left\{90^{\circ}>\mid \text { aro }- \text { arop } \mid>5^{\circ} \quad\right. \text { condition a } \\
& \left|a h_{1}\right|>89.9945^{\circ} \quad \text { condition b } \\
& \left|a h_{2}\right|>87^{\circ} \quad \text { condition } \mathrm{c} \\
& \left|a h_{i} h-a h_{i} r\right|>1^{\circ} \quad \text { condition d } \\
& \left|a h_{1}-a d h_{1} k-a d h_{1} e-a h_{1} r\right|>1^{\circ} \quad \text { condition e } \\
& \left|a h_{2}-a d h_{2} k-a d h_{2} e-a h_{2} r\right|>1^{\circ} \quad \text { condition } \mathrm{f}
\end{aligned}
$$

\subsection{Analysis of cause}

Be analyzed from the conditions described above, the following conclusions:

a) Under normal circumstances, the rotation angle of 0.5 seconds before and after the difference between the upper and lower housing gyroscope should be around 45, as long as the condition a, 
whether in low latitudes or at high latitudes static small circle of six school will start again, this behavior may be due to jump up and down the rotation angle of the gyro housing large numbers.

b) In the first six school calibration process, due to the continuous measurement of $a d h_{1} k$ and $a d h_{2} k$ require four rotations, the measurement channel can not compensate for the initial raw measurements $a d h_{1} k$ and $a d h_{2} k$; and the current gyro offset angle is generally about 25, so bring measured through changes in elevation can reach 50, plus other errors might satisfy the condition e, $\mathrm{f}$, resulting in six school fails.

c) In the course of the six schools, although the original measurement channel measurement compensates for height and angle error $a d h_{1} w$ and $a d h_{2} w$, but the condition e, f does not contain this error, when the carrier swing violently, repeater error increases, it will lead to Unable to meet the criterion of leaving school by six.

d) Condition $b$ means that when the angle is greater than the height of the gyro $89.9945^{\circ}$, six school reset, this condition will be satisfied at high latitudes.

e) Condition c refers to the moment when the gyro angle is greater than the height of $87^{\circ}$, six school reset, and this condition will meet near the equator.

\subsection{Calibration Analysis}

Through the calibration process it is ready to use $\Delta S_{i}, \Delta \delta_{i}$ measurement data, using the best method of estimating the initial position error filtering and gyro drift parameter error, and then solving the corresponding parameter channel to compensate error estimation method using Solver channel to channel differential measurement combination of angle and declination error $\Delta S_{i}, \Delta \delta_{i}$ is formed when the value of the error variable observables estimates calculated calibration process diagram shown in Figure 1.

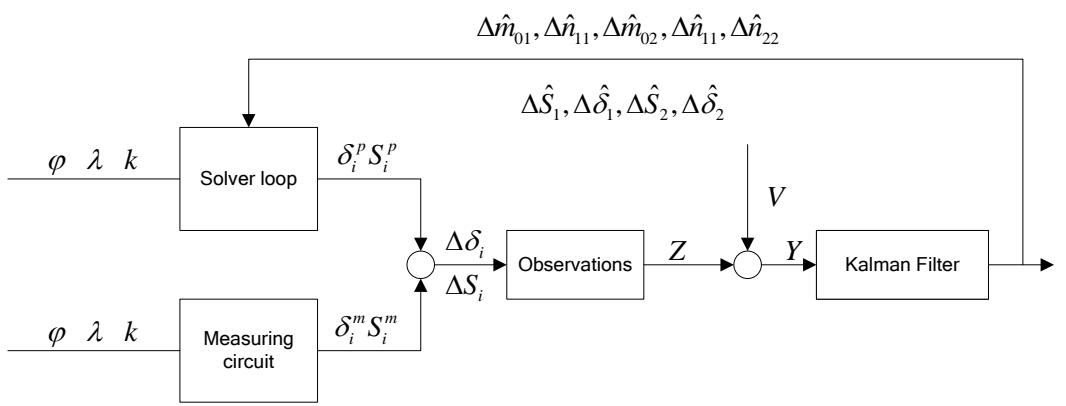

Figure 1. Schematic static calibration process

\subsection{Constraints of calibration phase}

During the electrostatic filter calibration reset program has nine criteria, as shown in the following equation.

$$
\left\{\begin{array}{l}
\Delta \hat{\delta}_{\mathrm{i}}>1^{\circ} \\
\Delta \hat{S}_{2}>1^{\circ} \\
\Delta \hat{m}_{0 \mathrm{i}}>0.01^{\circ} / h \\
\Delta \hat{n}_{1 \mathrm{i}}>0.1^{\circ} / h \\
\Delta \hat{n}_{22}>0.01^{\circ} / h
\end{array} \quad(\mathrm{i}=1,2)\right.
$$

From the electrostatic principle point of view, during system calibration work has two main channels, namely solver channels and measurement channels. Height declination angle error measurement channel raw measurements of repeater platform through a repeater of error due to the elevation angle error, gyro axis photoelectric sensor housing and the rotary shaft caused during calibration believed to be accurate. The Solver passage through six school determine declination angle when the initial value, the declination through the Earth's rotation caused by the angle of declination caused when the amount of change and the drift angle when the difference between the combination as a Kalman filter the observation equation, through the implementation of recursive estimation error variance. 
Through the above principle known to cause filter estimated value of the most direct reason is certainly that is larger than the observed two-channel declination angle and when the difference is relatively large, that estimate will filter value.

\section{Responses}

\subsection{Six school stage}

In order to make low latitudes six calibrations can be passed, the need to lower electrostatic gyro celestial corner of the initial value from the default value changed to $0{ }^{\circ} 5^{\circ}$. During calibration requirements while six ships sailing course security uniform, heading range $\pm 1.5^{\circ}$, the roll is greater than $3^{\circ}$, pitching less than $3^{\circ}$, six calibration operation should be selected in case the rotation angle is $0^{\circ}$ or $180^{\circ}$ when upper and lower gyro calibration, the calibration if there six gyroscopes ring counts frequently to zero, it is possible to adjust timely course, make the corresponding elevation gyro data curve smoothing, use upper and lower gyro "six calibration" points when the programs adopted.

\subsection{Calibration curve}

Under ideal conditions Electrostatic initiate and complete 48 hours calibration, the calibration process is affected by environmental factors, less interference, a calibration curve Z1, Z2, Z3 showed relatively smooth quadratic curve (Figure 2), Z2, Z3 performance for the first 24 hours Periodic changes in the laws of the curve, calibration data convergence after the end of 24 hours to more stable small value. Figure 3 is a calibration curve does not pass this time; Figure 4 is an electrostatic drift endurance by 24 hours prior to calibration calibration curve.

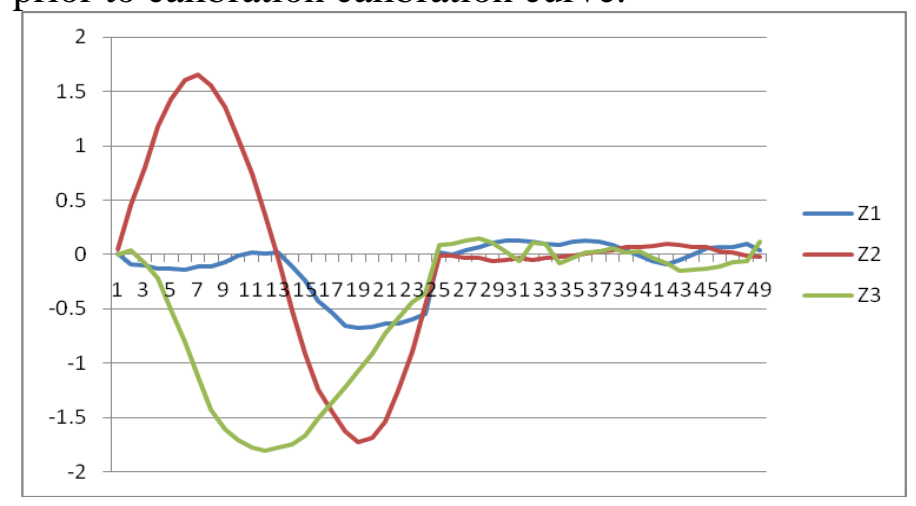

Figure 2. Standard calibration curve

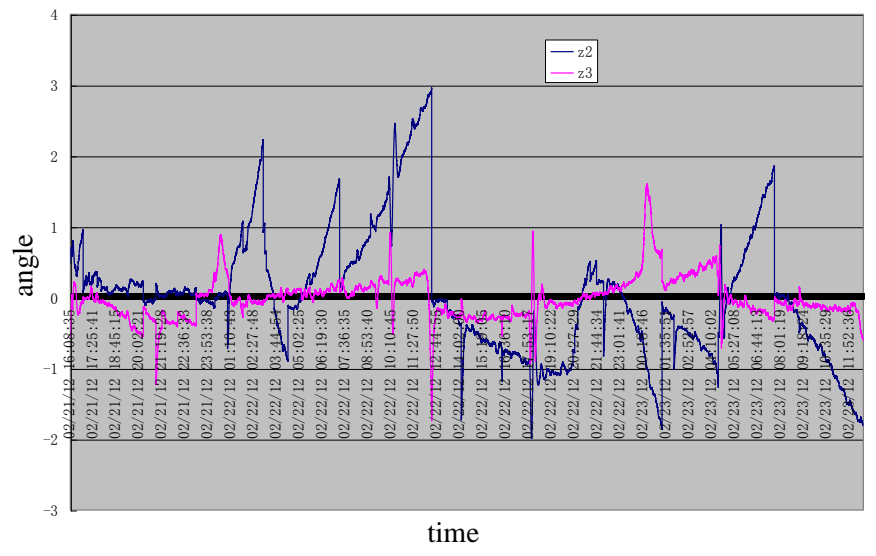

Figure 3. Calibration curve (data sampling 5 seconds / points, the same below) 


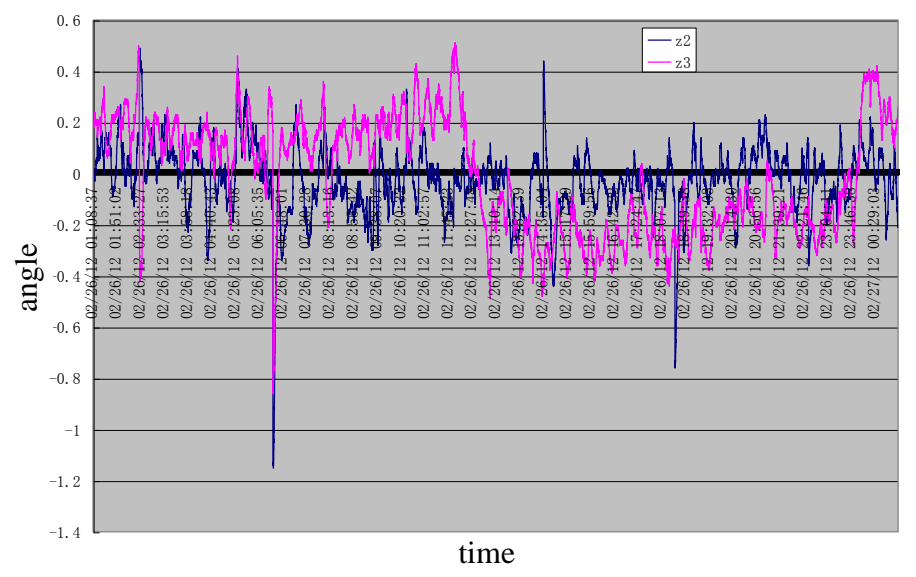

Figure 4. Electrostatic drift endurance 24 hours before calibration curve

Comparative Figures 3 and 4, the calibration curve can be found in Figure 4 significantly better in Figure 3, the first day and night ESG drift model parameter calibration results for $\mathrm{m}_{01}=-0.111133 \times 10^{-8}, \mathrm{~m}_{02}=-0.314989 \times 10^{-8}, \mathrm{n}_{22}=-0.497224 \times 10^{-8}$, meet $\left|\mathrm{m}_{01}, 02\right| \geq 0.4848 \times 10^{-7}$, and transferred to the navigation 12 hours after static heading accuracy $0.2^{\prime}$, maximum latitude error is 0.3 , longitude maximum error is 0.4 '.

\subsection{Drift Model}

ESG drift model coefficients magnitude representative of the accuracy of the static calibration process gyro drift, especially after static navigation 24 hours for gyro drift of magnitude higher, high static position and heading accuracy, good stability; magnitude drift coefficient the lower, the lower the static position and heading accuracy, stability worse. Under normal conditions, after the electrostatic turn navigation drift magnitude should be less than $10^{-8}$. Static in low latitudes navigation drift coefficient values may occur beyond the scope of this case should be written as the last drift coefficient value solver, and timely two-position correction, prevent the static data precision diverging result does not meet requirements. Figure 5 is a static position errors when a particular test, drift out of range curve, data is not available, by the drift to the last two solver and two pairs of normal position compensation operation, static position error in two days to stabilize, the electrostatic heading accuracy of 0.3 ', to meet the requirements.(Figure 6)

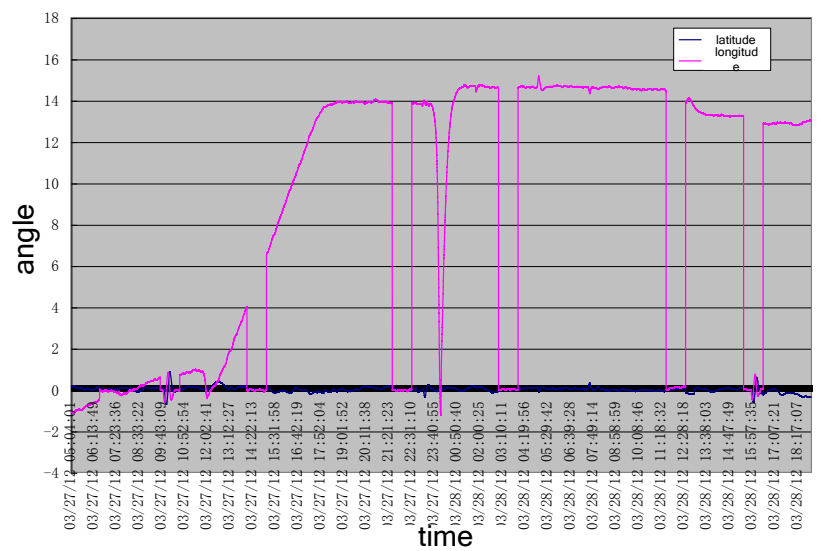

Figure 5. Static position error curve of exceeded m02 


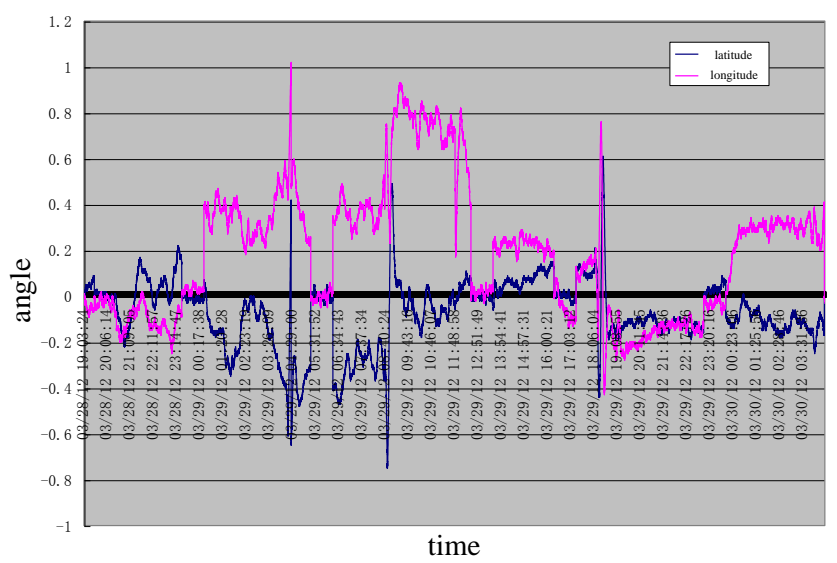

Figure 6. Static position error curve of changed m02 after 24 hours

\section{Summary}

From the time of starting the electrostatic prone to low latitudes delay by six schools and can not be calibrated by two questions, we analyze the reasons for the constraints and six school and calibration phase, static electricity can be obtained at present limited latitude outside latitude successful conclusion of the calibration start, alarm issue for the last uncaught drift coefficient prone to static electricity when running at low latitudes over the issue and the gyro gives the corresponding countermeasures, inspection by the task, effective measures for the future static at low latitudes start using the provided reference.

\section{References}

[1]. Pan Liang. Space tracking ship ship ship's position posture measurement technology [M]. Beijing: National Defense Industry Press, 2009.

[2]. Wu Junwei. ESG Monitor Technology [M]. Harbin: Harbin Engineering University Press, 2011.

[3]. Shipbuilding Heavy Industry 707. The new ship tracking ship inertial navigation systems pose their positions ESG monitor equipment maintenance manual[R].2006

[4]. Liu Xinming. ESG monitor sea "six school" method [J]. Chinese Inertial Technology, 2011, 19 (4): 399-402. 